\title{
Slow relaxation dynamics of clogs in a vibrated granular silo
}

\author{
B. V. Guerrero, ${ }^{1,}{ }^{*}$ L. A. Pugnaloni, ${ }^{2}$ C. Lozano, ${ }^{3}$ I. Zuriguel, ${ }^{1}$ and A. Garcimartín ${ }^{1}$ \\ ${ }^{1}$ Departamento de Física y Matemática Aplicada, Facultad de Ciencias, Universidad de Navarra, 31080 Pamplona, Spain \\ ${ }^{2}$ Departamento de Ingeniería Mecánica, Facultad Regional La Plata, Universidad Tecnológica Nacional, \\ CONICET, 1900 La Plata, Argentina \\ ${ }^{3}$ Fachbereich Physik, Universität Konstanz, Konstanz D-78457, Germany
}

(Received 8 February 2018; published 24 April 2018)

\begin{abstract}
We experimentally explore the vibration-induced unclogging of arches halting the flow in a two-dimensional silo. The endurance of arches is determined by carrying out a survival analysis of their breaking times. By analyzing the dynamics of two morphological variables, we demonstrate that arches evolve toward less regular structures and it seems that there may exist a certain degree of irregularity that the arch reaches before collapsing. Moreover, we put forward that $\sigma$ (the standard deviation of all angles between consecutive beads) describes faithfully the morphological evolution of the arch. Focusing on long-lasting arches, we study $\sigma$ calculating its two-time autocorrelation function and its mean-squared displacement. In particular, the apparent logarithmic increase of the correlation and the decrease of the mean-squared displacement of $\sigma$ when the waiting time is increased reveal a slowing down of the dynamics. This behavior is a clear hallmark of aging phenomena and confirms the lack of ergodicity in the unclogging dynamics. Our findings provide new insights on how an arch tends to destabilize and how the probability that it breaks with a long sustained vibration decreases with time.
\end{abstract}

DOI: 10.1103/PhysRevE.97.042904

\section{INTRODUCTION}

Gravity-driven flow of a dry dense granular medium outpouring through the bottom orifice of a silo is a representative case of discrete flows through a constriction [1-6]. In such systems, the flow may be temporarily or permanently interrupted by the formation of arches (or vaults) at the constriction [7-13]. The appearance and breakage of these blockages allow the distinction of three processes: continuous flow, clogging, and unclogging. They are intrinsically related to the clogging transition proposed in Ref. [4] and in a broader sense to the jamming transition of fragile matter [14-16]. The arches are sets of particles mutually stabilized by intergrain frictional forces and geometrical constraints. When they form, if all the kinetic energy of the system is dissipated, they last forever unless some external perturbation supplies the energy required to destabilize them.

Vibration is one of the most widely used perturbations to prevent or to break clogs. Regardless of whether the applied vibration is vertical [17-23] or if only the base on which the arch rests is vibrated horizontally [20,24], its aim is to allow the granular medium to explore different configurations and eventually reach one which may lead to the collapse of the arch. In this framework, robust clogs will be those that, in order to unclog them, it would require either a higher intensity or a longer duration of vibration. Therefore, in our study, the endurance of the arch is given by its ability to withstand the external perturbation instead of the ability to bear load.

In statistical terms, the stability of arches is characterized by the probability distribution (PDF) of breaking times $t_{b}$ (the

*Corresponding author: bguerrero.3@alumni.unav.es time that it takes to break an arch under a constant vibration level [25]). This distribution is broader than an exponential and displays a heavy tail compatible with a power law $t_{b}^{-\alpha}$, where $\alpha$ depends on the system conditions [4]. The existence or not of a well-defined average breaking time $\left\langle t_{b}\right\rangle$ depends on the $\alpha$ exponent. When $\alpha>2$ the mean converges and hence after a certain finite time the flow will always resume, so this situation is called an "unclogged state" [4]. Conversely, a "clogged state" refers to those cases in which $\alpha \leqslant 2$, where the average $\left\langle t_{b}\right\rangle$ diverges as the measurement time increases and is dominated by the occurrence of rare extreme clogging events. Then, through the convergence of the first moment of the PDF of $t_{b}$, the exponent $\alpha$ defines the aforementioned clogged-unclogged state transition.

Understanding the vibration-induced unclogging phenomenon can provide valuable insight on how the granular medium should be excited. A helpful approach has been to focus on the morphological features of clogging arches, neglecting other features of the granular medium. A useful variable when studying clogging in silos filled with spherical grains is the angle formed between consecutive grains of the arch $[8,20,26-28]$. Chiefly, the $\phi$ internal angle (of the closed polygon with vertices defined by the geometrical centers of the arch beads) subtended between the centers of a grain and its two nearest neighbors in the arch (see inset in Fig. 1) can be used to successfully predict the vibration intensity needed to resume the flow $[23,25,29]$. This fact can be understood in terms of a geometrical model based on mechanical equilibrium considerations $[20,23]$. When there exists at least one defect, i.e., the particle is hanging from above the equator due to static friction $(\phi \geqslant 180)$, the arch tends to be weaker and it will collapse sooner [25,29]. However, these assertions have only been proved for short-lasting arches, where the sensitivity to initial conditions is paramount. 

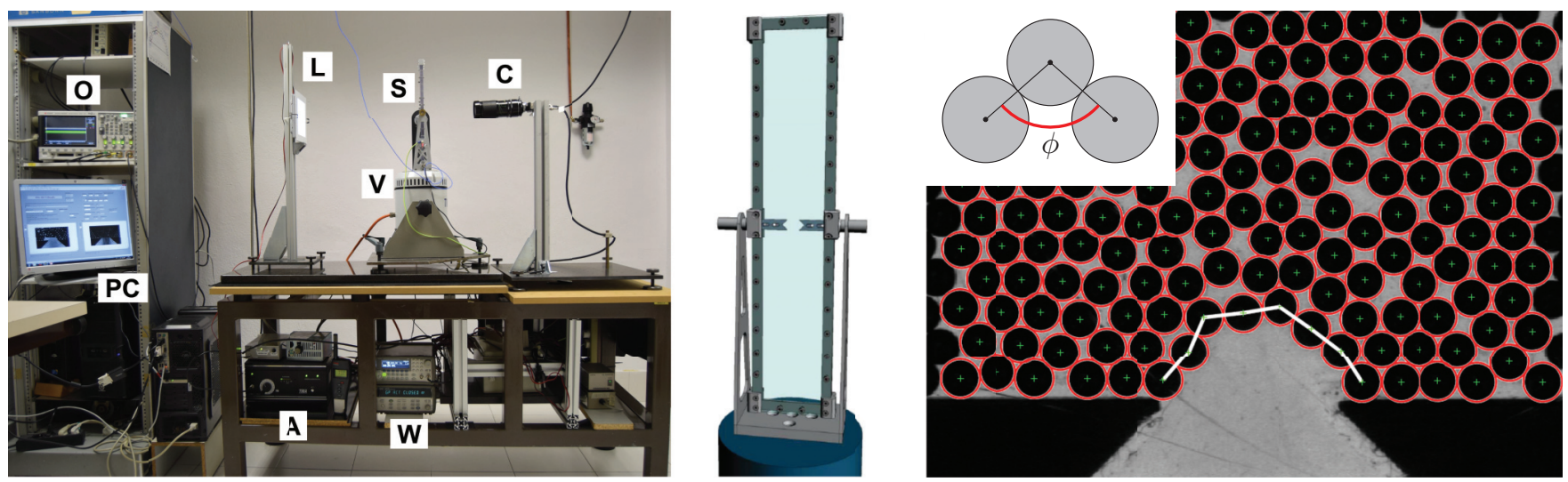

FIG. 1. Experimental setup and illustration of the image processing. Left: Photograph of the lateral view of the experimental setup. S, silo; W, waveform generator; A, amplifier; V, electromagnetic shaker; C, camera; L, LED lighting; O, oscilloscope; PC, computer. Center: Sketch of the frontal view of the silo. Right: A picture showing the result of image processing. The geometrical center of each bead was detected (green cross marks) and the arch has been identified (pink line). The $\phi$ angle among a bead and its two nearest neighbors is sketched in the inset.

Currently, it is not clear how long-lasting clogging arches evolve when they are submitted to vibration. In principle, they might tend to destabilize or to consolidate. Besides, there are many unknown issues about the dynamics of the unclogging process. For instance, despite the fact that we know that intergrain friction governs the dynamics of the arch stability $[29,30]$, it is still unclear if the dynamics are dominated by some abrupt events that lead to a catastrophic failure or if it follows instead a slow and smooth evolution. In this regard, a simple frictional model has shown some interesting resemblance with the dynamics of vibrated arches [31,32].

In order to address these aspects, we experimentally explore the relationship between the clog stability and some of its time-dependent morphological features. Our experiment consists on a two-dimensional silo filled with a monodisperse granular medium submitted to vertical vibrations, where the long-lasting clogging arches are identified and tracked along time. In particular, a low-intensity vibration has been employed to track small movements of the beads and then to clarify how the collapse or the consolidation of the arch is reached.

\section{EXPERIMENTAL SETUP}

In order to elucidate how vibration affects the arch dynamics, we have used a vibrated two-dimensional silo, which has been described previously $[23,25]$ and is depicted in Fig. 1 . The silo consists of two transparent polycarbonate sheets $(390 \mathrm{~mm}$ high $\times 80 \mathrm{~mm}$ wide), coated with indium tin oxide to prevent electrostatic charging. A $1-\mathrm{cm}$-wide and $1.2-\mathrm{mm}$-thick steel frame is sandwiched between the sheets. The granular medium consists of monodisperse nonmagnetic stainless steel spherical beads of diameter $d=1 \pm 0.01 \mathrm{~mm}$. Therefore, they can only form a monolayer inside the silo.

The silo is divided in two equal enclosures $(180 \mathrm{~mm}$ high $\times$ $60 \mathrm{~mm}$ wide) by two opposite movable metal flanges that form an orifice of variable length $D$. Based on previous knowledge $[8,20,25,33]$, we have chosen a fixed outlet size $D=4.4 d$ that, for the other conditions that will be explained below, has allowed us to get a large number of arches that collapse after sufficiently long time.
The silo works as an automated hourglass. The upper part of the container is filled with grains and the bottom chamber collects the discharged beads. A motor enables the silo to turn upside down around its horizontal axis. The silo is placed on top of a TiraVib 52100 electromagnetic shaker that delivers a sinusoidal vertical acceleration. This is generated by a waveform generator Agilent 33220A and it is amplified in order to achieve the desired vibration amplitude. The vibration level is characterized by the normalized maximum gravity acceleration: $\Gamma \equiv(2 \pi f)^{2} A / g$, where $g$ is the acceleration of gravity and $A$ and $f$ are the amplitude and the frequency of the oscillation, respectively. The measurement of the acceleration in the vertical direction and in the two transverse directions are carried out with a triaxial highsensitivity piezoelectric accelerometer (model PCB 356A22) placed on top of the shaker. The accelerometer's signal is digitized in a Keysight MSOX3024T oscilloscope. For all experiments the transverse acceleration is always less than $15 \%$ of the vertical acceleration. Since our aim is to achieve vibration-induced rearrangements of long-lasting arches, we have chosen a dimensionless maximum acceleration $\Gamma=0.6$, low enough to prevent local fluidization near of the arch zone (that could appear for $\Gamma \approx 1$ ) but also high enough to cause perturbations in the arches so that many of them break before $1200 \mathrm{~s}$. We set an oscillation frequency $f=105 \mathrm{~Hz}$, in order to have an oscillation amplitude $A \sim 13 \mu \mathrm{m}$, higher than the grains' roughness, which is a few microns or smaller after electron-microscope observations.

A standard charged coupled device (CCD) camera (model Hamamatsu C5405-51) placed in front of the backlighted silo captures the region near of the outlet at nearly 25 frames per second (including the time needed to save them to hard disk), with an exposure time of $1 / 125 \mathrm{~s}$. As the rate is not strictly constant, because of the time taken to write the files on the disk, we time-stamp the images. The experiment duration has been limited to $1200 \mathrm{~s}$. As the temporal resolution is about $0.04 \mathrm{~s}$, the dynamic range covers four orders of magnitude.

The experimental protocol is as follows. The silo is set in the vertical position with the grains in the upper chamber. Gravity-driven flow starts, and it stops when the silo is unfilled or a clog appears. If a clog occurs, we switch on a constant 
external vibration (with a fixed frequency and amplitude). When the arch breaks, or if $1200 \mathrm{~s}$ are reached without the arch breaking, the silo upper chamber is emptied by means of a stronger vibration. The silo is then turned and the next run starts.

Clogged and unclogged stages have been identified by comparing in real time each image with the previous one. During the clogged stage, the geometrical center of each bead is detected with a resolution of 0.5 pixels $(\sim 10 \mu \mathrm{m})$ with an in-house particle-tracking software, following the concepts provided by Shattuck and coworkers [34]. Once this is done, as shown in Fig. 1, the arch is identified by determining the lowest chain of interconnected beads spanning the silo outlet and then it is tracked along time.

\section{STATISTICS OF BREAKING TIMES}

The endurance of arches against a constant vibration is measured by $t_{b}$. Experimentally, this is calculated by subtracting the times corresponding to the last and the first images where the arch is identifiable. As we have set up an upper bound to the experiment duration, the breaking time data is right censored. From now on, according to the censoring condition, we will call "broken arches" those for which $t_{b}<1200 \mathrm{~s}$, whereas we will term "unbroken arches" those that remain unbroken at $1200 \mathrm{~s}$, when the experiment is stopped.

Survival analysis is useful to describe the statistics of the time that it takes for an event to occur, and in particular if data are censored [35]. In order to describe the breaking time data, we will calculate the survival function and the hazard ratio. If $t_{b}$ is a continuous random variable with probability distribution function $P(t)$, then the survival function $S(t)$ [also known as complementary cumulative probability distribution $\left.P\left(t_{b}>t\right)\right]$, is defined as:

$$
S(t) \equiv P\left(t_{b}>t\right) \equiv \int_{t}^{\infty} P\left(t^{\prime}\right) d t^{\prime},
$$

and gives the probability that an arch lasts longer than $t$. The hazard rate (also named hazard function) $h(t)$ is defined as a conditional failure rate within a certain time interval. It represents the instantaneous likelihood of an arch breakage per unit time, conditioned to the probability that an arch survives up to time $t$ :

$$
h(t) \equiv \lim _{\Delta t \rightarrow 0} \frac{P\left(t \leqslant t_{b}<t+\Delta t \quad \mid t_{b} \geqslant t\right)}{\Delta t},
$$

where the numerator is the conditional probability $P(\cdot \cdot \cdot)$ that the arch will break in the interval $[t, t+\Delta t)$ given that it has not broken before, and the denominator is the width of the time interval.

Note that $P(t), S(t)$, and $h(t)$ are mutually related and each of them contains the same information. In fact, if one of these is known, the others can be derived from this. In particular, the relationship existing between $S(t)$ and $h(t)$ is given by:

$$
h(t)=-\frac{d \ln [S(t)]}{d t} .
$$

However, when describing the statistics of the event of interest, $S(t)$ focuses on surviving whereas $h(t)$ focuses on failing, given the arch survived up to that time.

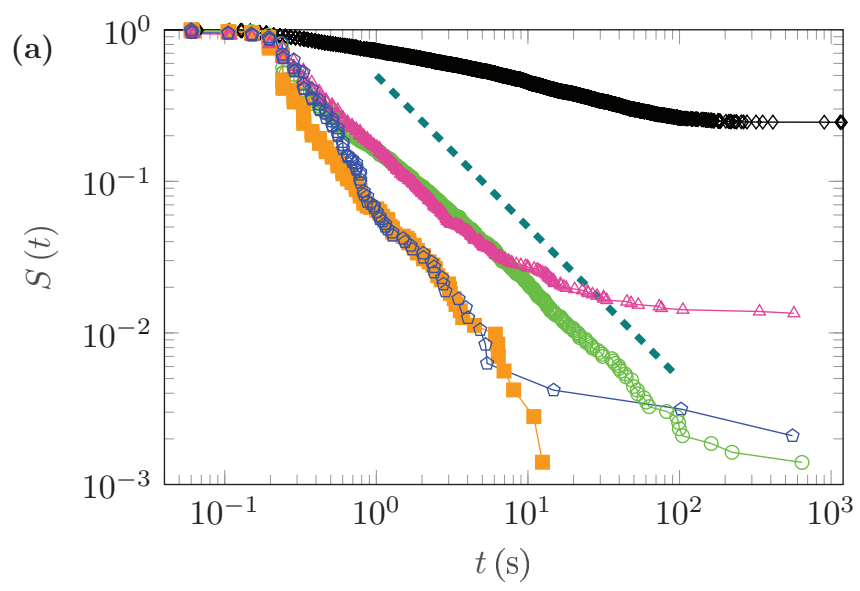

(b)

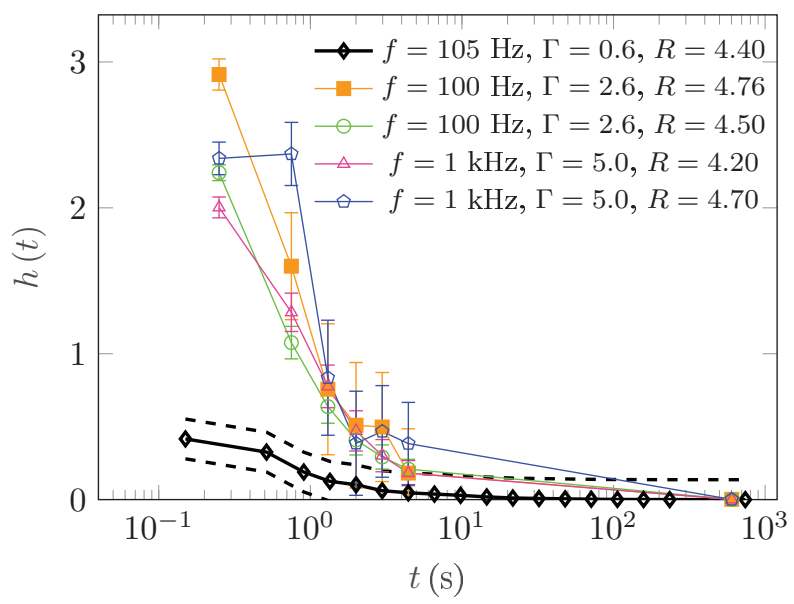

FIG. 2. Survival analysis of breaking time data $t_{b}$ for the current experiment $(\diamond)$ and for some of the experiments reported in Ref. [25] $(\boldsymbol{\square}, \square, \bigcirc, \triangle)$, as indicated in the legend. (a) Survival function (complementary cumulative distribution function) of $t_{b}$. The dashed line indicates the case $S(t) \sim t_{b}^{-\alpha+1}$ corresponding to $\operatorname{PDF}(t) \sim t^{-\alpha}$ with $\alpha=2$. (b) Hazard rate (with nonempty and unevenly spaced bins), where confidence intervals are shown as error bars for previously reported experiments [25] and as dashed lines for the current experiment.

For our current experiment, and some of the experiments previously reported in Ref. [25], survival and hazard functions were computed using nonparametric estimators, following the methods described in Ref. [36]. Figure 2(a) shows the survival functions of $t_{b}$ in a logarithmic scale where the heavy tails can be clearly appreciated. The main difference in our current data (black diamonds) with respect to previous ones [25] is due to the use of a low intensity vibration (lower than the fluidization limit $\Gamma=1$ ) that leads to an important proportion of unbroken arches $(25 \%)$. All survival functions behave as a power law followed by a tail that tends to flatten out for long times (see the region $t_{b}>100 \mathrm{~s}$ ). By performing a power-law fit to the logscaled survival plot of our current breaking time data (diamond symbols in Fig. 2), using the method described in Ref. [37], an exponent $\alpha \sim 1.2$ is obtained, meaning that the average $\left\langle t_{b}\right\rangle$ is undefined. Note that the presence of a plateau always implies that $\left\langle t_{b}\right\rangle$ cannot be defined, even if the power-law shape preceding the plateau had an exponent $\alpha>2$. In Ref. [25] the 

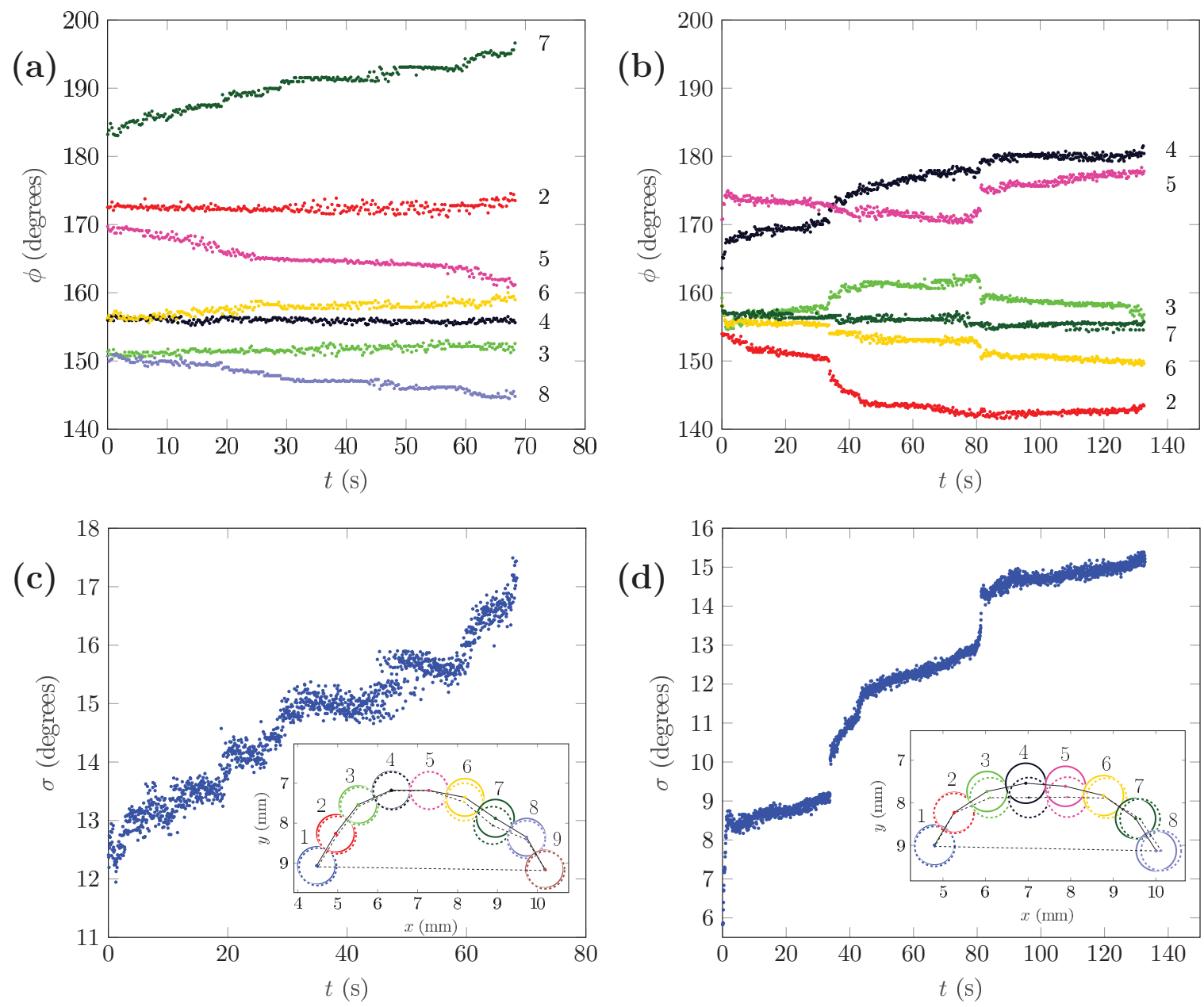

FIG. 3. Evolution of two long-lasting broken arches, [(a) and (c)] and [(b) and (d)], respectively. Upper panels show $\phi(t)$ for each grain, where $\phi$ is measured as sketched in Fig. 1. The lower panels show $\sigma(t)$ [see Eq. (4)]. The color and the number on the right side of each $\phi(t)$ curve indicates the bead of the arch (see the insets in lower figures). Insets show the position of each bead of the arch at two instants: just after starting the vibration (solid line) and just before the collapse (dashed line). In plots (a) and (b) data have been decimated by a factor five. Note that the $\phi$ range in (a) and (b) are the same, which is not the case for $\sigma$ in (c) and (d). Also note that the timescale is different for the two arches.

plateaus at the end in the log-scaled survival plot were related to highly regular arches where geometrical imperfections are very small. Recently, these plateaus have been rationalized in terms of stochastic models, where the arch breakage can be understood as the escape from an energy barrier [38] or in terms of a first passage process of a continuous time random walk [39].

In Fig. 2(b), we show the hazard function of the breaking times (with nonempty and unevenly spaced bins) for the same cases reported in Fig. 2(a). The estimated error bars were calculated with the formula for a binomial proportion confidence interval. It can be seen that the hazard rate is a monotonically decreasing function. This behavior can be compared with the survival of patients who are recovering from surgery. The death hazard after surgery decreases in time (under normal conditions) [35]. In our case, this means that the probability of breaking an arch with a sustained vibration decreases with time. In fact, after $100 \mathrm{~s}$ it approaches zero. Therefore, in industrial applications, the selection of intensity and duration of vibrations used to break clogs requires a complex balance. Low-intensity vibrations require less power but this may imply a rather long sustained excitation for very stable arches since the probability of breaking them decays with time.

\section{MORPHOLOGICAL REGULARITY OF THE ARCH}

To gain insight into how vibration affects the endurance of long-lasting arches, we obtained an ensemble of 4458 independent experimental realizations of clogging events. For each one of the realizations, the positions of every arch bead has been identified and tracked along time until breakage. Then, the angle $\phi(t)$ has been measured for all the beads of the arch except the two lowest beads, where $\phi$ is not defined.

We now show the dynamics of two arches that evolve differently and serve to illustrate the heterogeneity of the observed behavior. Figures 3(a) and 3(b) show the evolution of $\phi$ for each particle in these two arches. Both cases exhibit some intermittent large rearrangements of bead positions, in a fashion reminiscent of stick-slip dynamics but in a qualitaqualitatively different way. Figure 3(a) shows an arch that has lasted for a long time $(70 \mathrm{~s})$, despite having an initial defect. It looks 
(a)
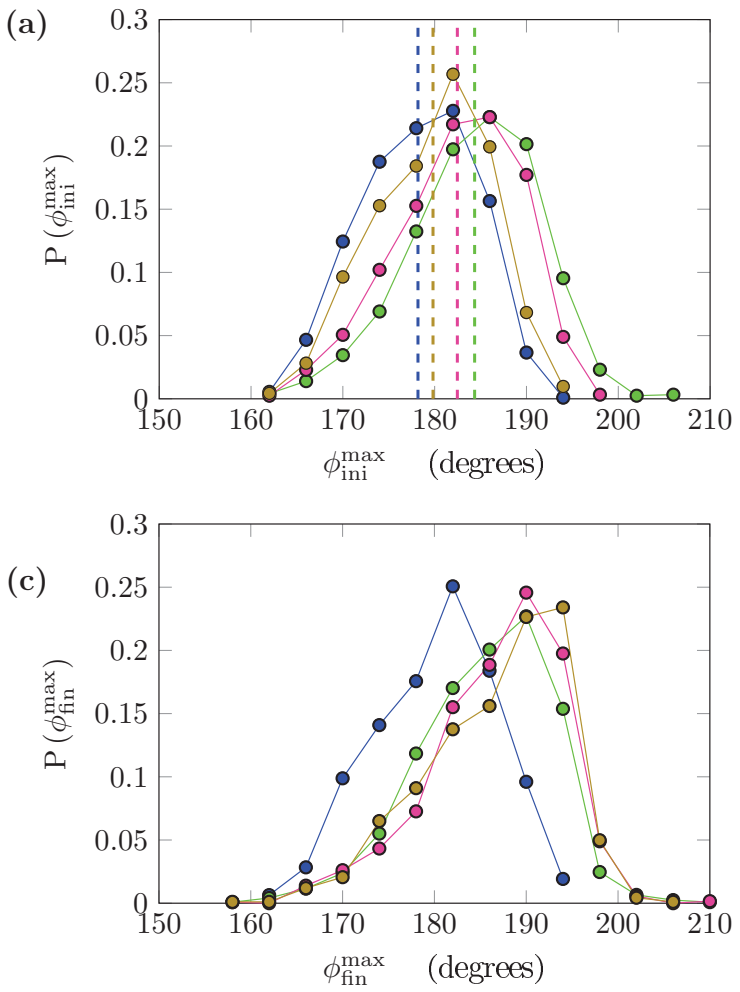
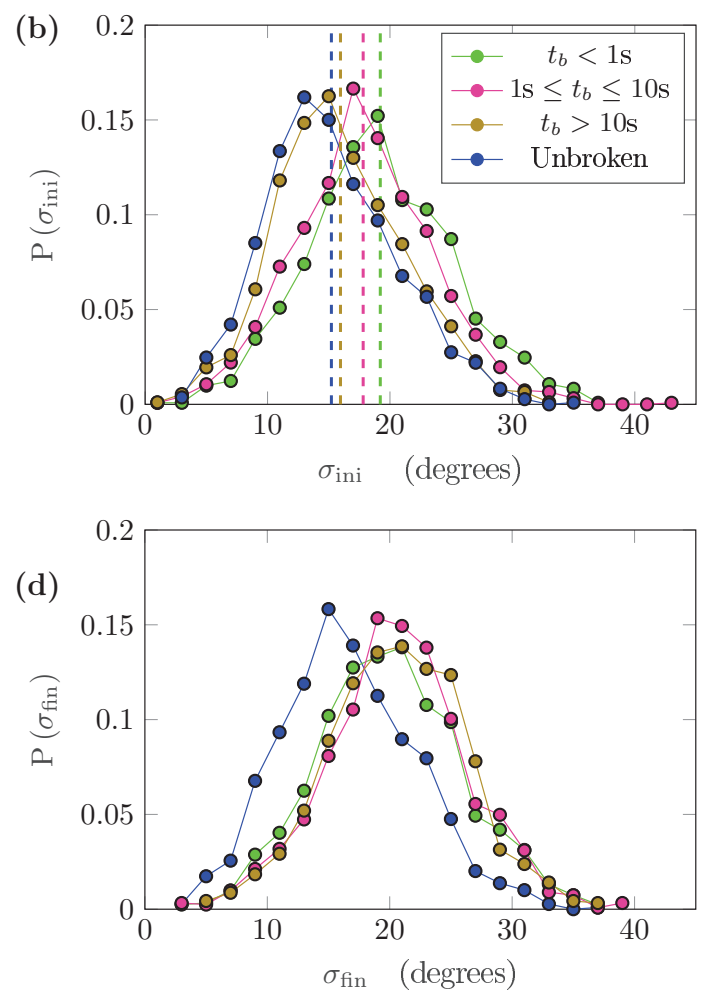

FIG. 4. Probability distribution function of (a) the maximum angle right after starting the vibration $\phi_{\text {ini }}^{\max }$, (b) $\sigma$ right after starting the vibration, (c) the maximum angle just before the collapse $\phi_{\mathrm{fin}}^{\max }$, and (d) $\sigma$ just before the collapse. Vertical dashed lines represent the average value of the distribution. Each color indicates the subset of arches (according to their breaking times), as is indicated in the legend.

as if the dynamics is fully governed by the "weakest link" [23] [i.e., the initial largest $\phi$ angle, dark green curve in Fig. 3(a)]. Apparently, the arch collapses when it reaches the maximum angle allowed by interparticle tangential friction, in agreement with the theoretical model proposed in Ref. [23]. Another remarkable feature in this case is that the arch evolves rather smoothly, although there are a few short events (appreciated as steps in the curves). Here the vibration weakens the arch and the angle $\phi$ of the the defect grows monotonically. The other case is shown in Fig. 3(b). The maximum angle decreases at the beginning and is overtaken by the second largest angle. After some time, at around $40 \mathrm{~s}$ and $80 \mathrm{~s}$, there are two sudden large rearrangements (which could be called burst in the framework of intermittent dynamics) that cannot be explained only in terms of the initial morphology of the arch. Later, the dynamics evolves comparatively smoothly until the arch collapses. The behavior observed in these two arches is similar to the reported by Merrigan and coworkers (see Fig. 2, Ref. [39]), where they calculated $\phi(t)$ by means of numerical simulations of a system equivalent to ours, although with a higher vibration level.

In the following, the morphological evolution from the initial to the final configuration will be analyzed statistically. In order to do this, we have classified the arches into unbroken arches (1093 arches) and three arbitrary subpopulations of broken arches, sorted according to their breaking time. The 1216 "short-lived" broken arches are the weakest, with a breaking time less than $1 \mathrm{~s}$; there are 1225 "intermediate" broken arches that last between 1 and $10 \mathrm{~s}$; and there are 923 "long-lasting" broken arches, taking more than $10 \mathrm{~s}$ to break.
In Fig. 4(a) the measured probability distribution $P$ of the initial maximum angle $\phi\left(\phi_{\mathrm{ini}}^{\max }\right)$ is shown for these four subpopulations. These histograms show that, on average, arches that last longer have lower maximum angles. However, the distributions are spread out and highly overlapping, and therefore $\phi_{\mathrm{ini}}^{\max }$ is not alone in determining the stability of an arch.

In Fig. 4(c) the measured probability distribution of the final maximum angle $\phi$ is shown $\left(\phi_{\mathrm{fin}}^{\max }\right.$ is measured either just before the breakage or when reaching $1200 \mathrm{~s}$ without breaking). The distributions corresponding to the broken and the unbroken arches subsets clearly differ and all the three broken arches subsets share practically the same skewed distribution regardless of their breaking time. In addition, note that for all broken arch families the distributions fall abruptly after $\phi_{c}=194 \pm 4$ degrees. This $\phi_{c}$ has been related to a stability threshold associated to the fully mobilized static friction [23].

In search of a variable that globally describes the stability of the arches, we have tested (i) the area of the polygon defined by the geometric centers of the spheres in the arch, (ii) the sum of all the angles $\phi$, and (iii) the magnitude of the vector defined by all the $\phi$ angles (as defined in Ref. [39]). However, after comparing the evolution of each of these variables with $\phi(t)$, we concluded that a suitable variable when describing the regularity of an arch is the standard deviation $\sigma$ of all angles $\left\{\phi_{2}, \ldots, \phi_{N-1}\right\}$ at a given instant:

$$
\sigma=\sqrt{\frac{\sum_{i=2}^{N-1}\left(\phi_{i}-\bar{\phi}\right)^{2}}{N-3}} \quad \text { (at time } t \text { ), }
$$


where $N$ is the number of beads in the arch and $\bar{\phi}$ is the average of $\phi$. Figures 3(c) and 3(d) show the evolution of $\sigma(t)$ for the same two arches shown in Figs. 3(a) and 3(b), respectively. It can be seen that $\sigma(t)$ captures well, with a single number, the overall behavior observed in $\phi(t)$ for each one of the beads. For example, the two abrupt movements [occurring at about $40 \mathrm{~s}$ and $80 \mathrm{~s}$ in Fig. 3(b)] are discernible in $\sigma(t)$ as evidenced from Fig. 3(d).

In Figs. 4(b) and 4(d) we have plotted $P\left(\sigma_{\text {ini }}\right)$ and $P\left(\sigma_{\text {fin }}\right)$ for the same arches as in Figs. 4(a) and 4(c). In Fig. 4(b), it is observed that the longer the breaking time, the lower the expected value of $\sigma$. In other words, long-lasting arches tend to be more regular. Again, $P\left(\sigma_{\text {ini }}\right)$ are spread out and highly overlapping just like $P\left(\phi_{\text {ini }}\right)$, so $\sigma_{\text {ini }}$ cannot serve to fully characterize the stability of an arch. In Fig. 4(d), the probability distribution of $\sigma$ at the end $P\left(\sigma_{\text {fin }}\right)$ is shown. Once more, as in Fig. 4(c), there is apparently a maximum degree of irregularity that the arch can withstand (on average) before breaking. Note that the distributions $P\left(\sigma_{\mathrm{fin}}\right)$ are not as sharp as $P\left(\phi_{\mathrm{fin}}^{\max }\right)$.

The morphological analysis based on initial and final configurations (both in terms of $\phi$ and $\sigma$ ) done in this section allows us to infer that the arches tend to become less regular when they are subjected to constant vibration. For the unbroken arches, although they also tend to become slightly less regular, they do not reach an unstable-enough configuration that triggers their destabilization.

\section{TWO-TIME DEPENDENT ANALYSIS}

In the previous section it has been shown that, apart from the intermittent burst dynamics related with some abrupt and large morphological rearrangements, the arch configuration evolves slowly until the arch collapses (see, e.g., Fig. 3). Inspired by the treatment carried out in Refs. [40,41] to characterize the slow relaxation of the compaction of vibrated granular media, we have calculated the two-time autocorrelation function of $\sigma$, defined as:

$$
C\left(t_{w}, \tau\right)=\frac{\left\langle\sigma\left(t_{w}+\tau\right) \sigma\left(t_{w}\right)\right\rangle-\left\langle\sigma\left(t_{w}+\tau\right)\right\rangle\left\langle\sigma\left(t_{w}\right)\right\rangle}{\sqrt{\operatorname{Var}\left(\sigma\left(t_{w}\right)\right) \operatorname{Var}\left(\sigma\left(t_{w}+\tau\right)\right)}},
$$

where $\langle\cdots\rangle$ denotes an ensemble average, $\operatorname{Var}(\sigma)$ is the variance of $\sigma$ at a certain time from the beginning of the vibration, and $t_{w}$ and $t_{w}+\tau$ are the two times where the autocorrelation is calculated. Usually, $t_{w}$ and $t_{w}+\tau$ are called the "waiting time" and the "observation time", respectively; and $\tau$ is the lag time. Note that $\sigma$ is an observable that measures the arch regularity [as defined in Eq. (4)] and is not intended as an order parameter. When the system is out of equilibrium, $C\left(t_{w}, \tau\right)$ depends on both $t_{w}$ and $t_{w}+\tau$, while at equilibrium it only depends on $\tau$.

In order to determine $C\left(t_{w}, \tau\right)$, we have arbitrarily chosen as our ensemble those broken arches that last more than $60 \mathrm{~s}$. We also tried with ensembles of independent sets of arches and we checked that our results are robust and do not depend on this choice. Figure 5 shows $C\left(t_{w}, \tau\right)$ for various $t_{w}$. Clearly, the autocorrelation depends on both $t_{w}$ and $\tau$, which reflects the intrinsic nonequilibrium dynamics. The larger $t_{w}$ is, the slower is the relaxation and the longer are the memory effects. This fact indicates that there is not time translation invariance and it constitutes a significant hallmark of aging phenomena

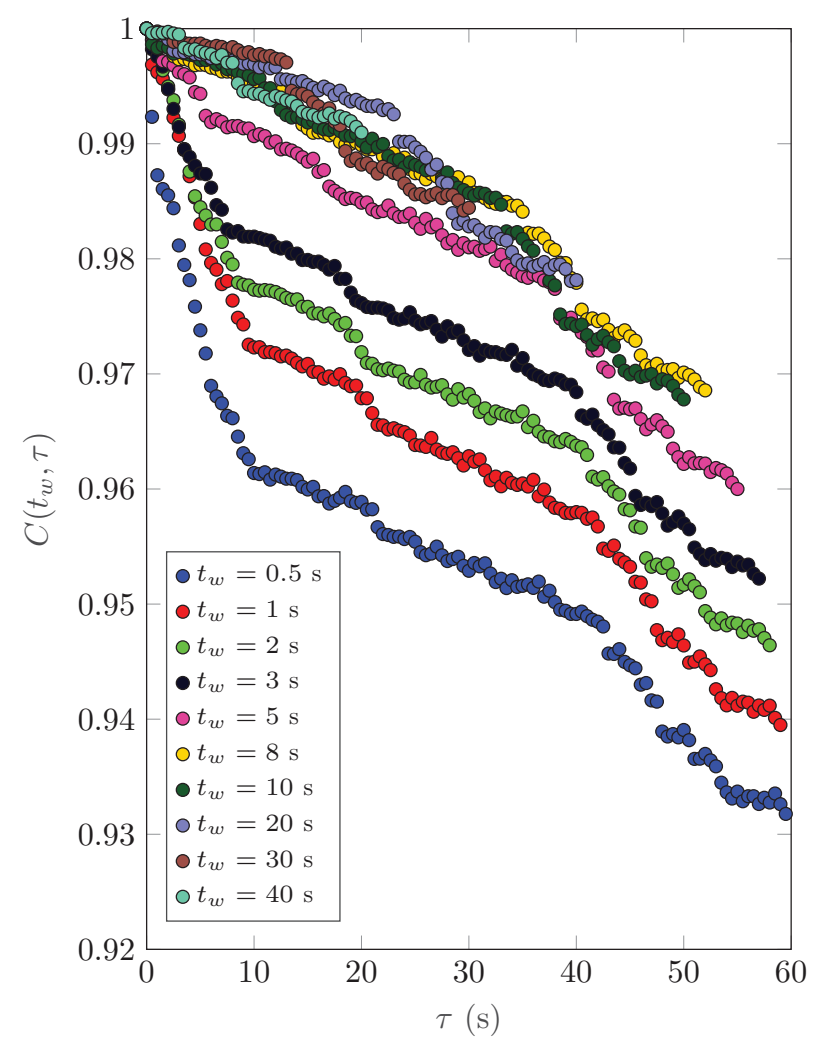

FIG. 5. Two-time $\sigma$ autocorrelation function $C\left(t_{w}, \tau\right)$ as a function of the lag time $\tau$ for various waiting times $t_{w}$.

[42]. This result is congruent with the recent numerical results in which the ergodicity breaking in the dynamics of arches submitted to vibrations has been shown [39]. As pointed out in Ref. [43], memory effects in a vibrated granular vanish only if the vibration is able to break each particle-particle contact in each vibration cycle. The nonconservative nature of the frictional interaction is the underlying mechanism to the aging of the system. However, a robust characterization of the observed aging is hampered by the short dynamic range (that covers two decades at the most), the experimental noise in $\sigma(t)$, and the small correlation decay. Indeed, the largest decay of correlations is less than $10 \%$, which is not surprising since the positions of the beads in the arch are highly correlated and they barely move before collapsing. Even within this small range for $C\left(t_{w}, \tau\right)$, it tends to converge for $t_{w} \gtrsim 10 \mathrm{~s}$, after which the dependence with $t_{w}$ is imperceptible. This convergence can be better appreciated with the aid of Fig. 6, where $C\left(t_{w}, \tau\right)$ is plotted versus $\left(t_{w}+\tau\right)$ (in logarithmic scale) for the same lag $\tau=10 \mathrm{~s}$ and different $t_{w}$. An apparent linear fit shows how the correlation increases logarithmically as $t_{w}$ increases, until an almost fully correlated state is reached for $t_{w} \gtrsim 10 \mathrm{~s}$.

Another feature of aging is the existence of a dynamical scaling with $\left(t_{w}+\tau\right) / t_{w}$ [42]. However, the negligible dependence of the autocorrelation with the waiting time for $t_{w}>10 \mathrm{~s}$ hinders a proper determination of such scaling. In any case, following an equivalent scaling to the used in Ref. [44], in Fig. 7 we plot $\ln \left[C\left(t_{w}, \tau\right)\right]$ vs $\ln \left[\left(t_{w}+\tau\right) / t_{w}\right]$. The dashed line suggests that there might exist a dynamical scaling behavior. Surely, there could be other possible scaling forms within this 


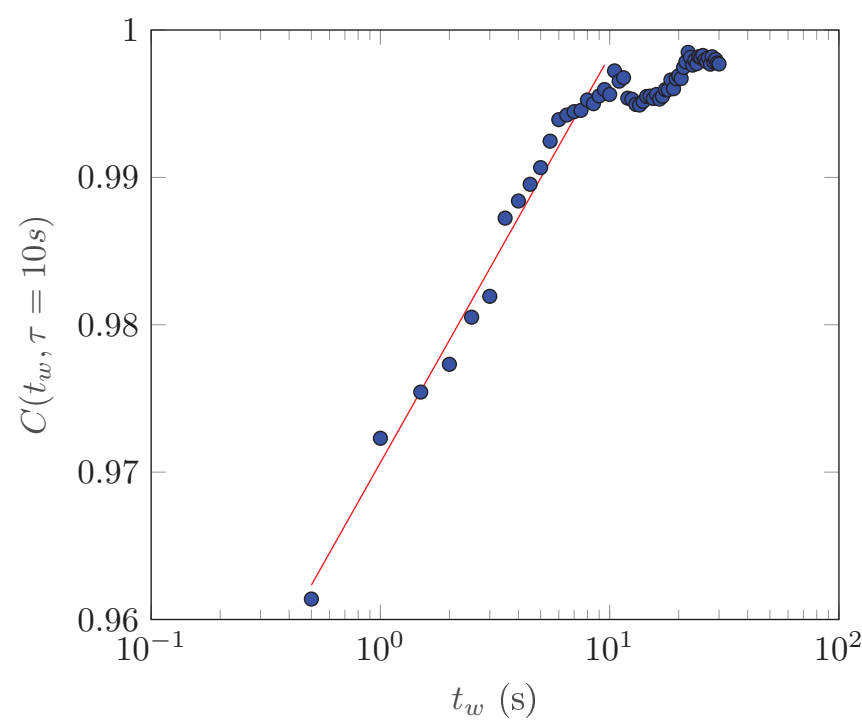

FIG. 6. Two-time $\sigma$ autocorrelation function $C\left(t_{w}, \tau\right)$ vs $t_{w}$, for a fixed lag time $\tau . t_{w}$ goes from 0.5 to $30 \mathrm{~s}$ in $0.5 \mathrm{~s}$ increments. Note the logarithmic scale of the $t_{w}$ axis. The straight line reveals the logarithmic increase of the correlation with time.

narrow time range (at most 2 decades). In any case, if such scaling were real, then it would be interesting to continue exploring the possibility of obtaining a time-dependent length scale based on a dynamic exponent derived from the particular scaling form [42].

Finally, let us focus in a complementary variable that evidences the aginglike effects in the unclogging dynamics: the (ensemble averaged) two-time mean-squared displacement of $\sigma$ :

$$
\left\langle\Delta \sigma^{2}\left(t_{w}, \tau\right)\right\rangle=\left\langle\left(\sigma\left(t_{w}+\tau\right)-\sigma\left(t_{w}\right)\right)^{2}\right\rangle
$$

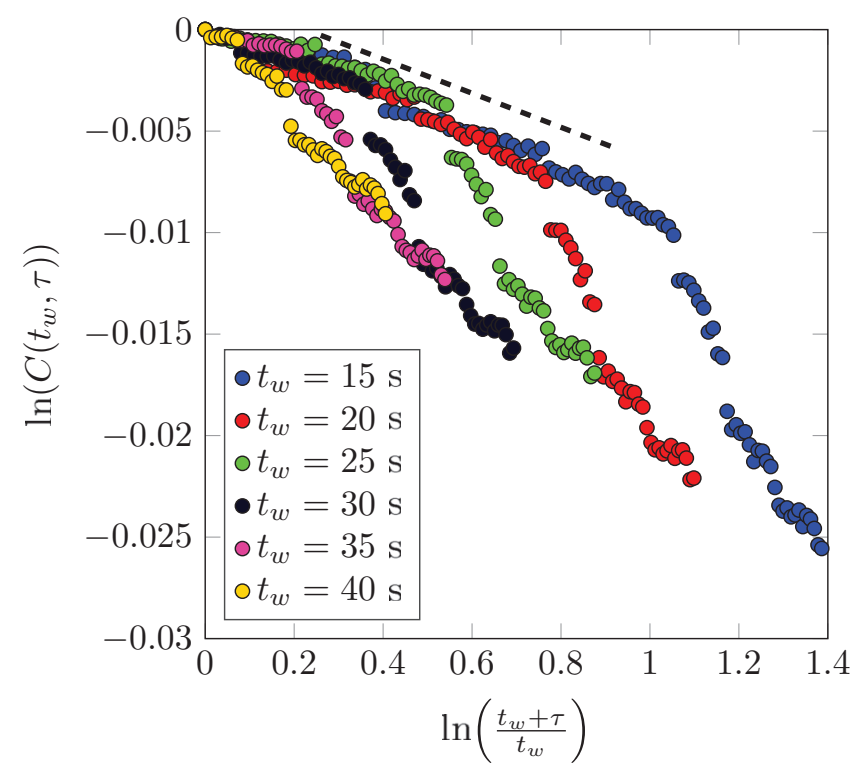

FIG. 7. Scaling plot for long $t_{w}$ of the two-time $\sigma$ autocorrelation function. The apparent collapse of data onto a single curve (dashed line) hints to the characteristic scaling of a phenomena undergoing aging effects.

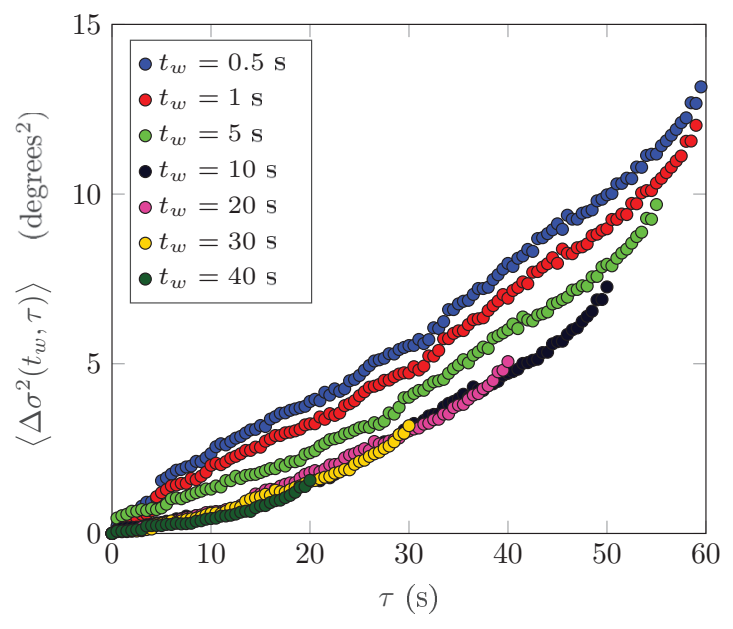

FIG. 8. Two-time mean-squared displacement of $\sigma$ for different $t_{w}$. The ensemble is composed of all broken arches lasting more than $60 \mathrm{~s}$.

where the angle brackets denote an ensemble average. Figure 8 shows $\left\langle\Delta \sigma^{2}\right\rangle$, recalling that the ensemble is composed of the broken arches lasting more than $60 \mathrm{~s}$. Again, we observe the tendency to a slower increase of $\left\langle\Delta \sigma^{2}\right\rangle$ as $t_{w}$ is increased; leading to values of $\left\langle\Delta \sigma^{2}\right\rangle$ which become almost insensitive to $t_{w}$ for $t_{w}>10 \mathrm{~s}$. This explicit dependence of $\left\langle\Delta \sigma^{2}\right\rangle$ on $t_{w}$ revalidates our finding that there is an aginglike phenomenon inherent to the unclogging dynamics of long-lasting arches. A deeper study of the scaling of $\left\langle\Delta \sigma^{2}\right\rangle$ vs $t$ and the implications of the aginglike effects on $\left\langle\Delta \sigma^{2}\right\rangle$ could help to understand the underlying process that leads to arches becoming less regular in terms of models of nonlinear diffusion [45]. Following this direction, it would also be desirable to determine the relationship between our mean-squared displacement and the one calculated in Ref. [39] (both time averaged and ensemble averaged). The information extracted from this may be key when examining the features of an associated stochastic process and could provide some insight into the local rheology in the surroundings of the outlet.

\section{CONCLUSIONS}

We have studied, for the first time in an experiment, the breaking dynamics of long-lasting arches spanning and blocking the outlet of a two-dimensional silo, when arches are submitted to a sustained external vibration of low constant intensity. The endurance of arches to external perturbation has been examined in terms of the breaking times through a survival analysis. We have also explored statistically the dynamics of the breaking process. First, we have based our analysis on two morphological variables which take into account the arch regularity: the angle $\phi$ between consecutive beads and the standard deviation $\sigma$ of $\{\phi\}$. We start by analyzing the configurations at the beginning and at the end of the clogging. The obtained results lead us to (a) reinforce the notion that the initial morphology (e.g., defects) of the arch affects the arch stability but is insufficient to determine the breaking time of the arch; (b) support the idea of the existence of a maximum stability threshold [23]; (c) assert that $\sigma$ is able to describe 
with a single number the overall evolution of the arch; and (d) confirm that vibration entails arches to evolve to less regular configurations that could eventually end up in a collapse.

Besides all the aforementioned statements, in order to understand how arches evolve to those less stable configurations, we have performed a dynamic study of the morphology of the arch in terms of $\sigma$. By calculating both the two-time autocorrelation function and the mean-squared displacement of $\sigma$, for different waiting times $t_{w}$, clear aging features were observed. In particular, an apparent logarithmic increment of the correlation when $t_{w}$ is increased has been found. Moreover, one possible dynamic scaling has been tested. Unfortunately, the experimentally accessible time range is not long enough to determine if such a scaling exists. This aginglike behavior is consistent with the lack of time translation invariance and the intrinsic nonequilibrium nature of granular systems.
The observed logarithmic relaxation (Fig. 5 to Fig. 8) may be reminiscent of the compaction process in vibrated granular media [41]. Indeed, from a fundamental point of view, it would be interesting to unveil if our results at a microscopic scale (namely, the grain scale) could be linked to descriptions at larger scales as in Ref. [46].

\section{ACKNOWLEDGMENTS}

We thank Luis F. Urrea for technical help and Bulbul Chakraborty for fruitful discussions. This work was funded by Ministerio de Economía y Competitividad (Spain) through Projects No. FIS2014-57325 and No. FIS2017-84631 MINECO/AEI/FEDER, UE Project. B.G. also thanks Asociación de Amigos de la Universidad de Navarra for a grant.
[1] R. M. Nedderman, Statics and Kinematics of Granular Materials (Cambridge University Press, Cambridge, 1992).

[2] J. Duran, Sands, Powders, and Grains: An Introduction to the Physics of Granular Materials (Springer, New York, 2000).

[3] S. Franklin, Handbook of Granular Materials (CRC Press, Taylor \& Francis Group, Boca Raton, FL, 2015).

[4] I. Zuriguel, D. R. Parisi, R. C. Hidalgo, C. Lozano, A. Janda, P. A. Gago, J. P. Peralta, L. M. Ferrer, L. A. Pugnaloni, E. Clément, D. Maza, I. Pagonabarraga, and A. Garcimartín, Sci. Rep. 4, 7324 (2014).

[5] I. Zuriguel, Pap. Phys. 6, 060014 (2014).

[6] X. Hong, M. Kohne, M. Morrell, H. Wang, and E. R. Weeks, Phys. Rev. E 96, 062605 (2017).

[7] S. Manna and H. Herrmann, Eur. Phys. J. E: Soft Matter Biol. Phys. 1, 341 (2000).

[8] K. To, P.-Y. Lai, and H. K. Pak, Phys. Rev. Lett. 86, 71 (2001).

[9] I. Zuriguel, L. A. Pugnaloni, A. Garcimartín, and D. Maza, Phys. Rev. E 68, 030301 (2003).

[10] I. Zuriguel, A. Garcimartín, D. Maza, L. A. Pugnaloni, and J. M. Pastor, Phys. Rev. E 71, 051303 (2005).

[11] C. C. Thomas and D. J. Durian, Phys. Rev. E 94, 022901 (2016).

[12] J. Koivisto and D. J. Durian, Phys. Rev. E 95, 032904 (2017).

[13] A. Ashour, S. Wegner, T. Trittel, T. Börzsönyi, and R. Stannarius, Soft Matter 13, 402 (2017).

[14] M. E. Cates, J. P. Wittmer, J.-P. Bouchaud, and P. Claudin, Phys. Rev. Lett. 81, 1841 (1998).

[15] A. J. Liu and S. R. Nagel, Nature (London) 396, 21 (1998).

[16] H. Peter, A. Libal, C. Reichhardt, and C. J. O. Reichhardt, arXiv:1712.03307.

[17] M. L. Hunt, R. C. Weathers, A. T. Lee, C. E. Brennen, and C. R. Wassgren, Phys. Fluids 11, 68 (1999).

[18] C. R. Wassgren, M. L. Hunt, P. J. Freese, J. Palamara, and C. E. Brennen, Phys. Fluids 14, 3439 (2002).

[19] K. Chen, M. B. Stone, R. Barry, M. Lohr, W. McConville, K. Klein, B. L. Sheu, A. J. Morss, T. Scheidemantel, and P. Schiffer, Phys. Rev. E 74, 011306 (2006).

[20] J. R. Valdes and J. C. Santamarina, Can. Geotech. J. 45, 177 (2008).

[21] C. Mankoc, A. Garcimartín, I. Zuriguel, D. Maza, and L. A. Pugnaloni, Phys. Rev. E 80, 011309 (2009).

[22] P. Wen, N. Zheng, J. Nian, L. Li, and Q. Shi, Sci. Rep. 5, 9880 (2015).
[23] C. Lozano, G. Lumay, I. Zuriguel, R. C. Hidalgo, and A. Garcimartín, Phys. Rev. Lett. 109, 068001 (2012).

[24] K. To and H.-T. Tai, Phys. Rev. E 96, 032906 (2017).

[25] C. Lozano, I. Zuriguel, and A. Garcimartín, Phys. Rev. E 91, 062203 (2015)

[26] K. To and P.-Y. Lai, Phys. Rev. E 66, 011308 (2002).

[27] A. Longjas, C. Monterola, and C. Saloma, J. Stat. Mech.: Theory Exp. (2009) P05006.

[28] A. Garcimartín, I. Zuriguel, L. A. Pugnaloni, and A. Janda, Phys. Rev. E 82, 031306 (2010).

[29] R. C. Hidalgo, C. Lozano, I. Zuriguel, and A. Garcimartín, Granular Matter 15, 841 (2013).

[30] A. Ashour, T. Trittel, T. Börzsönyi, and R. Stannarius, Phys. Rev. Fluids 2, 123302 (2017).

[31] B. Blanc, L. A. Pugnaloni, and J.-C. Géminard, Phys. Rev. E 84, 061303 (2011).

[32] B. Blanc and J.-C. Géminard, Phys. Rev. E 88, 022201 (2013).

[33] T. Masuda, K. Nishinari, and A. Schadschneider, Phys. Rev. Lett. 112, 138701 (2014).

[34] M. D. Shattuck, Particle tracking [http://gibbs.engr.ccny.cuny. edu/technical/Tracking/ChiTrack.php].

[35] D. Kleinbaum, Survival Analysis: A Self-Learning Text (Springer, New York, 2012).

[36] J. P. Marques de Sá, Applied Statistics: Using SPSS, Statistica, $M A T L A B$, and $R$ (Springer, Berlin, 2007).

[37] A. Clauset, C. R. Shalizi, and M. E. J. Newman, SIAM Rev. 51, 661 (2009).

[38] A. Nicolas, A. Garcimartín, and I. Zuriguel, Phys. Rev. Lett. (to be published) [arXiv:1711.04455].

[39] C. Merrigan, S. K. Birwa, S. Tewari, and B. Chakraborty, Phys. Rev. E (to be published) [arXiv:1711.00964].

[40] M. Nicodemi and A. Coniglio, Phys. Rev. Lett. 82, 916 (1999).

[41] J. Talbot, G. Tarjus, and P. Viot, Eur. Phys. J. E: Soft Matter Biol. Phys. 5, 445 (2001).

[42] M. Henkel, Non-Equilibrium Phase Transitions (Springer, Dordrecht, 2010).

[43] P. A. Gago, D. Maza, and L. A. Pugnaloni, Pap. Phys. 8, 080001 (2016).

[44] S. Majumder and W. Janke, Phys. Rev. E 93, 032506 (2016).

[45] D. A. Stariolo, Phys. Rev. E 55, 4806 (1997).

[46] G. Lumay and N. Vandewalle, Phys. Rev. Lett. 95, 028002 (2005). 